\title{
Physiotherapy and Weaning From Prolonged Mechanical Ventilation
}

\author{
Annia F Schreiber, Piero Ceriana, Nicolino Ambrosino, Alberto Malovini, and Stefano Nava
}

\begin{abstract}
BACKGROUND: Patients undergoing prolonged mechanical ventilation represent up to $15 \%$ of all patients requiring weaning from mechanical ventilation. Although recent guidelines have recommended including physiotherapy early during mechanical ventilation to speed the process of weaning, only indirect evidence supporting the use of physiotherapy is available for patients receiving prolonged mechanical ventilation. The aim of our study was to evaluate the effects of a physiotherapy program in subjects requiring prolonged mechanical ventilation and the correlates of successful weaning. METHODS: A retrospective analysis was performed on 1,313 consecutive patients admitted to a weaning unit over a 15 -y period to be liberated from prolonged mechanical ventilation. Subjects underwent a program of intensive physiotherapy organized in $\mathbf{4}$ incremental steps (1-4) and were analyzed according to the steps achieved ( $>2$ steps or $\leq 2$ steps). The rate of successful weaning was recorded, and possible predictors were considered. The 15 -y period of observation was divided into 3 consecutive 5-y intervals. RESULTS: Out of 560 subjects undergoing final analysis, $349(62.3 \%)$ were successfully weaned. The weaning success rate was significantly greater in subjects attaining $>2$ steps than in subjects who attained $\leq 2$ steps $(72.1 \%$ vs $55.9 \%$, respectively, odds ratio $=2.04,95 \% \mathrm{CI}=1.42-2.96, P<.001$ ). Stepwise logistic regression analysis showed that achievement of $>2$ physiotherapy steps was the main predictor of successful weaning (odds ratio $=2.17$, 95\% $\mathrm{CI}=1.48-3.23, P<.001)$. Underlying disease was also a predictor of successful weaning. The overall rate of successful weaning decreased, and the median duration of weaning increased, during the period of observation. CONCLUSIONS: Our data support the inclusion of physiotherapy in the management of patients requiring prolonged mechanical ventilation. Key words: weaning; prolonged mechanical ventilation; physiotherapy; pulmonary rehabilitation; respiratory intermediate intensive care unit; critically ill subjects. [Respir Care 2019;64(1):17-25. () 2019 Daedalus Enterprises]
\end{abstract}

\section{Introduction}

Weaning, the process of withdrawing mechanical ventilation, begins as soon as the pathology leading to intu-

\footnotetext{
Drs Schreiber and Ceriana are affiliated with the Respiratory Intensive Care Unit and Pulmonary Rehabilitation Unit, Istituti Clinici Scientifici Maugeri IRCCS, Istituto Scientifico di Pavia, Italy. Dr Amrosino is affiliated with the Istituti Clinici Scientifici Maugeri IRCCS, Istituto Scientifico di Montescano IRCCS, Italy. Dr Malovini is affiliated with the Laboratory of Informatics and Systems Engineering for Clinical Research, Istituti Clinici Scientifici Maugeri IRCCS, Istituto Scientifico di Pavia, Italy. Dr Nava is affiliated with Alma Mater University, Deparment of Clinical, Integrated and Experimental Medicine (DIMES), Respiratory and Critical Care Unit, S. Orsola-Malpighi Hospital, Bologna, Italy.
}

Dr Schreiber presented a version of this paper was presented at the ERS International Congress, held September 26-30, 2015, in Amsterdam. bation is considered sufficiently controlled to allow a return to spontaneous breathing. ${ }^{1,2}$ Weaning accounts for up to $50 \%$ of the total duration of mechanical ventilation. ${ }^{1-3}$ Up to $80 \%$ of patients admitted to an ICU can be successfully weaned in the first attempt, whereas a smaller proportion fail the first spontaneous breathing trial (SBT) and may require up to $3 \mathrm{SBT}$ or up to $7 \mathrm{~d}$ from the first attempt to achieve successful weaning. ${ }^{4-6}$ These categories

\footnotetext{
The authors have disclosed no conflicts of interest.

Correspondence: Annia F Schreiber, Respiratory Intensive Care Unit and Pulmonary Rehabilitation Unit, Istituti Clinici Scientifici Maugeri IRCCS, Istituto Scientifico di Pavia, Via S Maugeri 10, 27100 Pavia, Italy. E-mail: annia.schreiber@icloud.com.
}

DOI: $10.4187 /$ respcare. 06280 
have been previously defined as simple, difficult, and prolonged weaning, respectively. ${ }^{2}$

\section{See the Related Editorial on Page 112}

After patients are clinically stable, those who require prolonged mechanical ventilation can be transferred to a weaning unit, a step-down unit, or a long-term facility. These units have a weaning success rate of up to $60 \%$ for patients with prolonged mechanical ventilation. ${ }^{7,8}$ Better outcomes have been reported when a program of intensive physiotherapy is used. ${ }^{9-11}$ Approximately $25 \%$ of patients requiring prolonged mechanical ventilation develop early muscle weakness. ${ }^{12}$ Evidence of benefits from early mobilization and physiotherapy has expanded through randomized, controlled trials and systematic reviews. ${ }^{13-19}$

Accordingly, we conducted an analysis of all subjects admitted to our weaning unit over a 15-y period who were on prolonged mechanical ventilation and underwent an intensive physiotherapy program. We assessed the effect of the physiotherapy program on the weaning success rate, as well as the correlates of weaning success and changes in outcomes over time.

\section{Methods}

Data from subjects consecutively admitted to the weaning unit of the Istituti Clinici Scientifici Maugeri IRCCS, Pavia, Italy, were prospectively collected between September 2000 and December 2014 and retrospectively analyzed at the end of this period. Subjects gave written informed consent to the anonymous handling of their data for scientific purposes, and the study was approved by the Institutional Ethics Committee (359 CEC).

\section{Setting and Subjects}

Our weaning unit consists of 7 fully monitored beds equipped for noninvasive and invasive mechanical ventilation, which are located within the rehabilitative respiratory ward of a multidisciplinary rehabilitation hospital. A 24-h on-duty doctor is available, the nurse-to-patient ratio is $1: 4$, and the physiotherapist-to-patient ratio is $1: 7$. Patients admitted to our weaning unit can be invasively (via tracheostomy) or noninvasively ventilated, or may need only continuous monitoring and intensive nursing care. Patients are transferred either from ICUs of other hospitals or from other wards within our hospital. Our Italian national health system refunds a maximal hospital stay of up to 5 weeks.

\section{QUICK LOOK}

\section{Current knowledge}

Patients undergoing prolonged mechanical ventilation (PMV) represent up to $15 \%$ of those requiring weaning from mechanical ventilation. Muscle weakness is a major factor contributing to difficult weaning in these patients. Recent guidelines recommend using physiotherapy early in mechanical ventilation to speed the process of weaning, but this recommendation remains to be confirmed for patients who require PMV.

\section{What this paper contributes to our knowledge}

In this 15-y analysis of subjects undergoing PMV, an intensive physiotherapy program was useful in the management of these subjects. Subjects who achieved more physiotherapy steps showed higher weaning success. Attaining $>2$ physiotherapy steps was the main predictor of successful weaning. However, confounding factors such as age and underlying disease also influence weaning, thus making it impossible to draw conclusions regarding the effect of physiotherapy on weaning.

\section{Physiotherapy Program}

Soon after admission to the weaning unit, all patients undergo an intensive physiotherapy program until the end of their hospital stay, regardless of weaning success. We use a fully validated and previously adopted 4-step early mobilization program involving levels of increasing difficulty, which is similar to that applied to ICU patients. ${ }^{20-22}$ The steps are defined according to (1) the ability to maintain a sitting position on the edge of the bed and to perform cycling against resistance in bed; (2) the ability to maintain a sitting position in a chair and regain standing posture; (3) the ability to start active transfer from bed to chair and to walk with the aid of a rollator and physiotherapists; and (4) the achievement of walking autonomy, with or without the aid of a stick and/or a person. Progressing from one step to the next depends on limb muscle strength improvement and the ability to sustain greater work loads. In addition to physical training, the physiotherapy program includes optimization of airway clearance and humidification, when needed.

The physiotherapy program in step 1 entailed passive arm and leg motion twice per day for $6 \mathrm{~d} /$ week. In particular, it included flexion and extension, supination and pronation, internal and external rotation, abduction and adduction for each joint. In step 2, active-assisted and active exercises were introduced, with the addition of sitting in a 
chair for 20 min twice per day, as well as exercises involving transition from a sitting to a standing position. Step 3 included the addition of active transfer to a chair and walking with the aid of a rollator and physiotherapists. Two walking sessions per day were scheduled with the goal of progressively increasing the distance, as tolerated by the subject. In step 4 , scheduled walking sessions were performed with a stick and/or a person by the subject's side.

In the last $5 \mathrm{y}$ of the study, the physiotherapy program was implemented with a Motomed machine (Reck Medizintechnik, Germany) and Standing equipment (Standy Electro Wing, Ormesa, Italy). The Motomed is a motor-driven, software-controlled movement therapy system incorporating a leg exerciser suitable for patients who are able to cycle against resistance as well as for those only able to move their limbs passively through motor assistance from the machine. With Motomed, manual physiotherapy sessions were replaced with machine-assisted sessions with two 20-min cycling sessions, initially in a passive manner at a fixed pedaling rate of 20 cycles/min. When subjects were able to cycle actively, the cycling sessions were divided into 2 rounds of $10 \mathrm{~min}$ or into more intervals if needed, with the goal of progressively increasing the resistance as tolerated by the subject. Standing equipment is a device designed to help bedridden patients achieve and maintain a standing position; it was used as a supplementary device in the phase of regaining standing posture.

\section{Weaning Protocol}

For the purposes of our study, prolonged mechanical ventilation was defined as at least $6 \mathrm{~h} / \mathrm{d}$ of mechanical ventilation for 21 consecutive days. ${ }^{23}$ Weaning from prolonged mechanical ventilation includes protocol-based daily monitoring of readiness for a SBT, ${ }^{24}$ followed by increasingly longer periods of SBT until the achievement of permanent spontaneous breathing.

Typically, medical treatment consists of bronchodilator therapy and monitoring of patient nutritional status, cardiovascular profile, metabolic state, and water balance, according to clinical needs and the baseline disease.

\section{Data Collection and Analysis}

Subjects' age, sex, unit of provenance, underlying disease, Simplified Acute Physiologic Score II (SAPS II) and relevant comorbidities were collected upon admission. ${ }^{25}$ Comorbidities were grouped into 3 major categories: metabolic (diabetes, obesity, endocrinopathies), cardiovascular (systemic and pulmonary artery hypertension, cardiac failure and ischemia, atrial fibrillation, and arrhythmias), and oncological (previous history of cancer without evidence of active disease). The observation period was di- vided into 3 consecutive 5-y periods (2000-2004, 20052009, and 2010-2014).

For data analysis, subjects were divided into 4 groups and analyzed according to the underlying disease responsible for the respiratory failure and prolonged mechanical ventilation. Group 1 included chronic pulmonary diseases (eg, COPD, bronchiectasis, restrictive lung and thoracic diseases); Group 2 included acute hypoxemic respiratory failure (eg, pneumonia, pulmonary embolism, refractory hypoxia, ARDS, cardiogenic pulmonary edema); Group 3 included postsurgery respiratory failure; and Group 4 included neuromuscular diseases.

\section{Measurements}

All subjects underwent assessment of muscular force at admission $\left(\mathrm{T}_{0}\right)$ and at discharge $\left(\mathrm{T}_{1}\right)$ on the basis of the Medical Research Council scale, ${ }^{26}$ which grades muscular strength from 0 (no muscle contraction visible or felt) to 5 (normal power). In the event of score improvement during weaning unit stay, the difference $\left(\Delta \mathrm{T}_{1}-\mathrm{T}_{0}\right)$ was calculated.

\section{Outcomes}

For the purpose of the study, successful weaning was defined as a 7-d ventilator-free period after a successful SBT. ${ }^{23}$ The primary outcome was the weaning success rate according to the achieved physiotherapy step. The secondary outcomes were the correlates of weaning success. The tertiary outcomes were the analysis of changes of different trends (achieved physiotherapy steps and weaning rate and duration) over the three 5-y periods.

According to the physiotherapy steps, subjects were divided into 2 categories: subjects achieving $\leq 2$ steps, and subjects achieving $>2$ steps. Further data included length of weaning unit stay, discharge destination, SAPS II, comorbidities, and weaning unit mortality.

\section{Statistical Analysis}

The distribution of quantitative variables was described by median (interquartile range [IQR]), whereas categorical variable distribution was described by count and frequency (\%). The Kruskal-Wallis test and the Wilcoxon rank-sum test were used to test for statistically significant differences in terms of quantitative and ordinal variables by year range. Pearson's chi-square test and 2-sided Fisher exact test were applied to test for any associations between categorical variables, as appropriate. The 2-sided Cochrane Armitage trend test and the Jonckheere-Terpstra test were applied to test for the presence of statistically significant trends in terms of categorical variables and distributions of quantitative variables over time. Univariate logistic regres- 


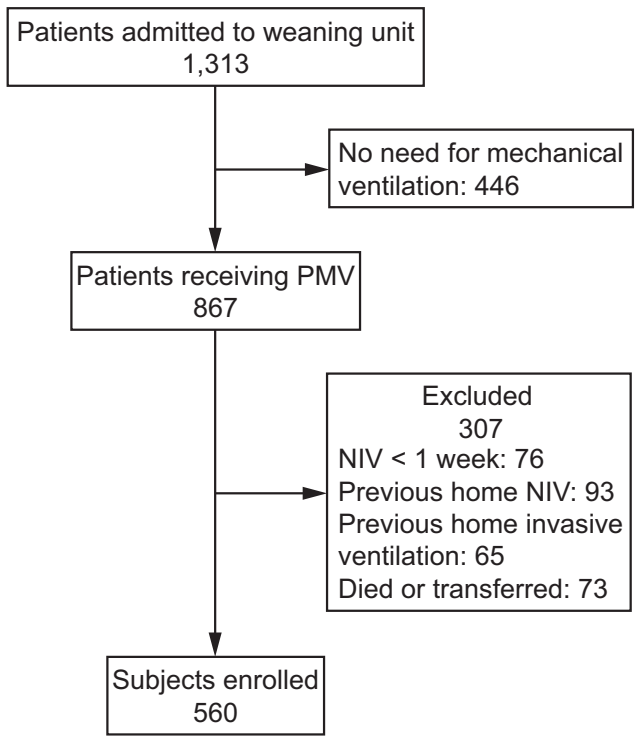

Fig. 1. Flow chart. PMV = prolonged mechanical ventilation; NIV = noninvasive ventilation.

sion was used to assess the effect of each variable on the probability of successful weaning and $>2$ rehabilitative steps. Stepwise multivariate logistic regression was also applied to identify the most informative subset of variables jointly modulating the probability of the outcomes of interest. Statistical procedures were performed with R software (version 3.4.1, R Development Core Team, Vienna, Austria). ${ }^{27}$ The Jonckheere-Terpstra test was implemented in the R package clinfun. Stepwise logistic regression was performed with the step function (R package stats).

\section{Results}

\section{Demographics and Subject Characteristics on Admission}

During the observation period, 1,313 consecutive patients were admitted (median age 71 y [IQR $=63-77]$, $57.96 \%$ male, $64.74 \%$ admitted from ICUs, mean SAPS II score $27.2 \pm 4.4)$. Sixty-six percent of patients $(867 / 1,313)$ were receiving mechanical ventilation on admission, but only 560 subjects were included in the final analysis (Fig. 1). All subjects required prolonged invasive mechanical ventilation (range 23-41 d) via tracheostomy. The distribution of different baseline diseases in subjects over time is reported in Figure 2. The prevalence of Group 1 subjects decreased over time, whereas that of Group 4 significantly increased. The overall median weaning unit stay was $30 \mathrm{~d}$ $(\mathrm{IQR}=25-35.5)$, and the mortality rate was $13.8 \%$.

\section{Primary Outcome}

Out of 560 subjects, $349(62.3 \%)$ were successfully weaned. Of the remaining 211 unweaned subjects (37.7\%),
$37(17.5 \%)$ were discharged with long-term invasive mechanical ventilation, 60 subjects $(28.5 \%)$ were transferred to long-term facilities with invasive mechanical ventilation, $73(34.6 \%)$ died, and 41 subjects (19.4\%) were decannulated and switched to noninvasive ventilation.

The weaning success rate was significantly greater in subjects attaining $>2$ steps than those who attained $\leq 2$ steps (72.1\% vs $55.9 \%$, odds ratio $=2.04,95 \% \mathrm{CI}=1.42-2.96$, $P<.001$ ) (Table 1).

Stepwise logistic regression showed that the achievement of $>2$ steps was the main predictor of successful weaning (Table 2) and was associated with a 2 -fold increase in the probability of the same outcome (odds ratio $=2.17,95 \% \mathrm{CI}=1.48-3.23, P<.001)$.

The muscular strength Medical Research Council score increased in all subjects, but the $\Delta \mathrm{T}_{1}-\mathrm{T}_{0}$ was significantly greater in weaned subjects $(1.15 \pm 0.68$ vs $0.48 \pm 0.6$ in weaned and unweaned subjects, respectively, $P<.001$ ). The mean SAPS II score did not differ between subjects with successful or failed weaning $(26.7 \pm 3.8$ vs $27.1 \pm 4.1$, respectively).

\section{Secondary Outcomes}

From the results of univariate analyses (Table 1), underlying disease was the only variable significantly associated with the achievement of $>2$ steps $(P<.001)$, whereas multivariate stepwise logistic regression analysis revealed that age and baseline disease jointly modulated the probability of attaining $>2$ rehabilitative steps (Table 2). Specifically, the likelihood of being able to reach $>2$ steps decreased as a function of age (odds ratio $=0.98,95 \% \mathrm{CI}=$ $0.97-0.99, P<.001)$ and was greater in Group 1, Group 2, and Group 3 (odds ratio $=5.83,95 \% \mathrm{CI}=3.94-8.78, P<$ .001 ; odds ratio $=3.74,95 \% \mathrm{CI}=2.54-5.59, P<.001$; and odds ratio $=4.62,95 \% \mathrm{CI}=3.10-6.99, P<.001$, respectively), as compared to Group 4.

Stepwise logistic regression (Table 2) indicated that underlying disease was a predictor of successful weaning. In particular, subjects in Group 2 and Group 3 showed the greatest likelihood of being successfully weaned, as compared with Group 4 (odds ratio $=3.28,95 \% \mathrm{CI}=1.85$ $5.89, P<.001$ [Group 2 vs Group 4] and odds ratio $=2.38$, $95 \% \mathrm{CI}=1.35-4.25, P=.003$ [Group 3 vs Group 4], respectively), whereas the outcomes of subjects in Group 1 were not significantly different from those in Group 4 . Comorbidities were not found to influence weaning outcome or achievement of higher physiotherapy steps.

\section{Tertiary Outcome}

During the stay in the weaning unit, $43.4 \%$ of subjects achieved $>2$ steps; however, this rate changed significantly over the study years, showing a tendency toward 


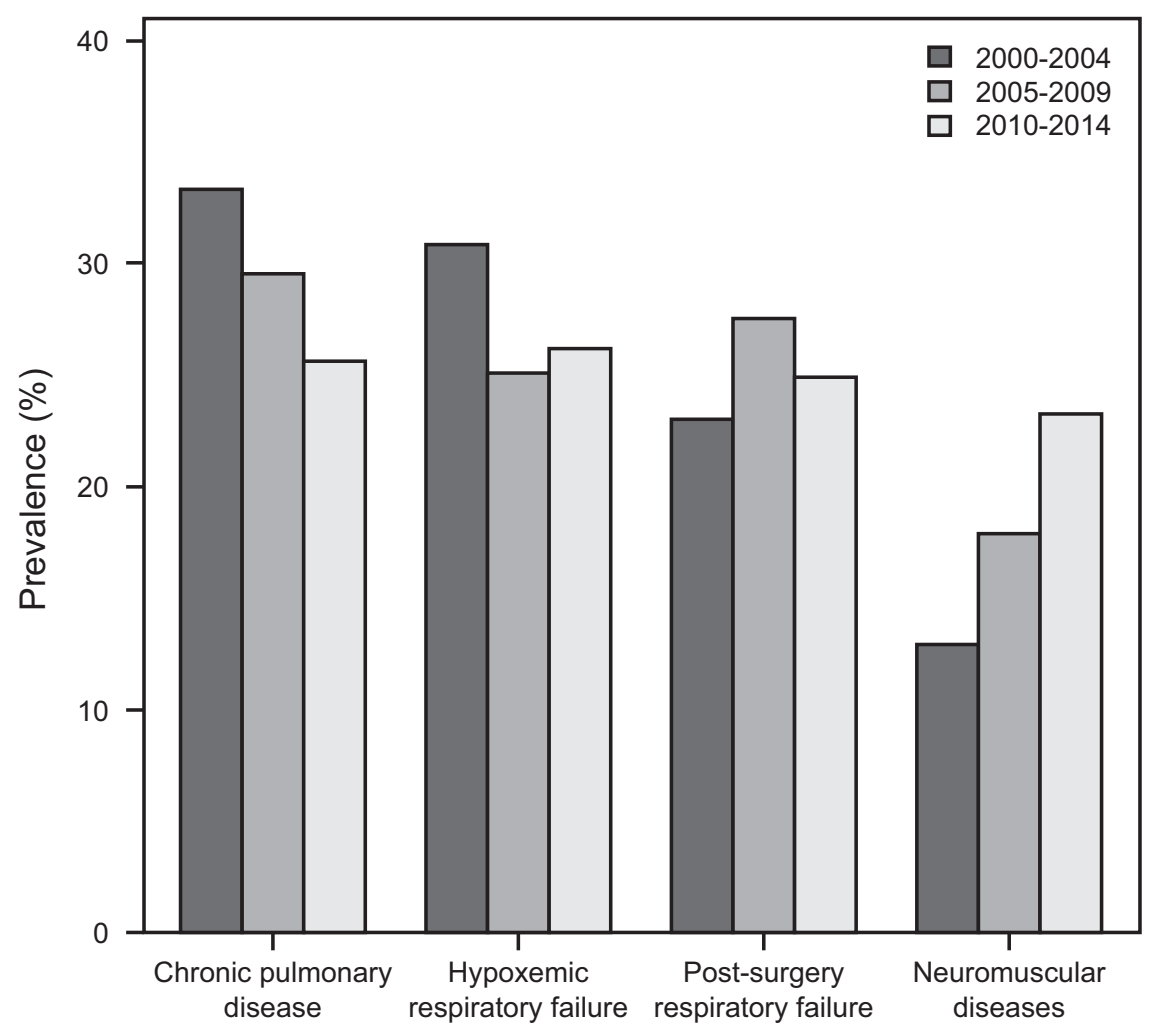

Fig. 2. Prevalence of subject disease groups over time. Group 1 includes COPD, bronchiectasis, and restrictive lung and thoracic diseases $(P=.048)$. Group 2 includes pneumonia, pulmonary embolism, refractory hypoxia, ARDS, and cardiogenic pulmonary edema $(P=.15)$. Group 3 includes postsurgical respiratory failure $(P=.32)$. Group 4 includes neuromuscular diseases $(P<.001)$.

achievement of a higher median step $(P=.002)$. In particular, a significant increase in the percentage of subjects reaching $>2$ steps was found over the years $(27.12 \%$, $48.51 \%$, and $50.84 \%$ for the periods $2000-2004$, 20052009, and 2010-2014, respectively, $P<.001$ ).

The weaning success rate decreased in the period 2010 2014, as compared with 2000-2004 and 2005-2009 (47.85\% vs $71.1 \%, P<.001$, and $47.85 \%$ vs $66.07 \%$, $P<.001$ respectively), and the median duration of weaning increased over the study years $(P=.002)$ (Fig. 3).

\section{Discussion}

Our study supports the inclusion of physiotherapy in the management of patients requiring prolonged mechanical ventilation. Such patients represent $5-15 \%$ of all patients requiring weaning from mechanical ventilation and are placed in the category of difficult weaning. ${ }^{2}$ Muscle weakness is a major contributing factor, together with malnutrition, chronic cardiac and respiratory disease, depression, anxiety, and previous delirium. ${ }^{19}$ Studies have suggested that a comprehensive rehabilitation strategy is desirable to facilitate the weaning program. ${ }^{28}$

Our 15-y analysis yielded 3 main findings. First, we found a higher successful weaning rate in subjects who achieved a higher physiotherapy step ( $>2$ steps); second, we noted a strong positive predictive value of a higher physiotherapy step regarding the probability of weaning, as identified through stepwise logistic regression; and third, we observed a greater gain in muscular strength in subjects who were successfully weaned. Age and baseline disease also appeared to influence weaning, whereas comorbidities did not. The severity index at admission appeared to be similar in weaned and not weaned subjects.

We suggest that physiotherapy positively influences the process of weaning in patients who require prolonged mechanical ventilation. Although recent guidelines recommend including physiotherapy in the acute care setting after the initiation of mechanical ventilation to speed the process of weaning, only indirect evidence is available supporting the use of physiotherapy for subjects under prolonged mechanical ventilation. ${ }^{11}$ In a study by Nava in $1998,{ }^{20}$ intensive physiotherapy was found to improve dyspnea, respiratory muscle, and lower limb force in a group of subjects admitted to a weaning unit and undergoing mechanical ventilation. Almost $10 \mathrm{y}$ later, Carlucci and coworkers $^{29}$ found that, in a group of difficult-to-wean subjects admitted to a weaning unit and undergoing intensive physiotherapy, the recovery of adequate inspiratory muscle force was the major determinant of weaning suc- 


\section{Physiotherapy and WeAning From PMV}

Table 1. Results From Univariate Tests

\begin{tabular}{|c|c|c|c|c|}
\hline \multicolumn{5}{|c|}{ Successful Weaning } \\
\hline Variable & Yes & No & Odds Ratio $(95 \% \mathrm{CI})$ & $P$ \\
\hline$n(\%)$ & $348(62.25) \dagger$ & $211(37.75)$ & NA & NA \\
\hline Age, y & $73(64-78)$ & $72(66-77)$ & $1(0.98-1.01)^{*}$ & .88 \\
\hline \multicolumn{5}{|l|}{ Gender } \\
\hline Female & $150(62.24)$ & $91(37.76)$ & Reference value & NA \\
\hline Male & $198(62.26)$ & $120(37.74)$ & $1(0.71-1.41)$ & $>.99$ \\
\hline \multicolumn{5}{|l|}{ Disease group } \\
\hline 1 & 77 (49.04) & $80(50.96)$ & $1.18(0.69-2.05)$ & .55 \\
\hline 2 & $121(75.16)$ & $40(24.84)$ & $3.72(2.11-6.63)$ & $<.001$ \\
\hline 3 & $115(70.55)$ & $48(29.45)$ & $2.94(1.69-5.18)$ & $<.001$ \\
\hline 4 & $35(44.87)$ & $43(55.13)$ & Reference value & NA \\
\hline \multicolumn{5}{|c|}{ Rehabilitative steps } \\
\hline$\leq 2$ & $190(55.88)$ & $150(44.12)$ & Reference value & NA \\
\hline$>2$ & $158(72.15)$ & $61(27.85)$ & $2.04(1.42-2.96)$ & $<.001$ \\
\hline \multicolumn{5}{|c|}{ Rehabilitative Step } \\
\hline Variable & $>2$ Steps & $\leq 2$ Steps & Odds Ratio (95\% CI) & $P$ \\
\hline$n(\%)$ & $569(43.40)$ & $742(56.60)$ & NA & NA \\
\hline Age, y & $71(61-77)$ & $72(64-77)$ & $0.99(0.98-1)^{*}$ & .11 \\
\hline \multicolumn{5}{|l|}{ Gender } \\
\hline Female & $240(43.64)$ & $310(56.36)$ & Reference value & NA \\
\hline Male & $329(43.23)$ & $432(56.77)$ & $0.98(0.79-1.23)$ & .88 \\
\hline \multicolumn{5}{|l|}{ Disease group } \\
\hline 1 & $203(53.14)$ & $179(48.86)$ & $4.25(2.96-6.18)$ & $<.001$ \\
\hline 2 & $157(44.10)$ & $199(55.90)$ & $2.95(2.05-4.32)$ & $<.001$ \\
\hline 3 & $158(47.73)$ & $173(52.27)$ & $3.42(2.36-5.02)$ & $<.001$ \\
\hline 4 & $51(21.07)$ & $191(78.93)$ & Reference value & NA \\
\hline \multicolumn{5}{|c|}{$\begin{array}{l}\text { Data are presented as } n(\%) \text { except for age, which is mean (range). } \\
* \text { Variation per year. } \\
\dagger \text { One individual was excluded due to missing values. } \\
\text { NA = not applicable }\end{array}$} \\
\hline
\end{tabular}

Table 2. Results From Multivariate Logistic Regression

\begin{tabular}{|c|c|c|}
\hline \multicolumn{3}{|c|}{ Dependent Variable: Successful Weaning } \\
\hline Independent variables & Odds Ratio $(95 \% \mathrm{CI})$ & $P$ \\
\hline \multicolumn{3}{|l|}{ Disease group } \\
\hline 1 & $0.90(0.51-1.59)$ & .71 \\
\hline 2 & $3.28(1.85-5.89)$ & $<.001$ \\
\hline 3 & $2.38(1.35-4.25)$ & .003 \\
\hline Rehabilitative steps: $>2$ & $2.17(1.48-3.23)$ & $<.001$ \\
\hline \multicolumn{3}{|c|}{ Dependent Variable: Rehabilitative Steps: $>2$} \\
\hline Independent variables & Odds Ratio (95\% CI) & $P$ \\
\hline Age, y & $0.98(0.97-0.99)^{*}$ & $<.001$ \\
\hline \multicolumn{3}{|l|}{ Disease Group } \\
\hline 1 & $5.83(3.94-8.78)$ & $<.001$ \\
\hline 2 & $3.74(2.54-5.59)$ & $<.001$ \\
\hline 3 & $4.62(3.10-6.99)$ & $<.001$ \\
\hline
\end{tabular}

cess, thus providing indirect evidence of a close link between physiotherapy and weaning from mechanical ventilation. Because successful weaning implies a restoration of the load/force balance, improvement of muscular force is a mandatory step, together with the optimization of the load component (ie, adequate bronchodilation, optimal airway humidification, and clearance of secretions). Similar results, albeit limited to a program of inspiratory muscle training, have been reported by Martin and coworkers ${ }^{30}$ in a group of difficult-to-wean subjects.

The actual cause by which difficult weaning is improved by physiotherapy (eg, lung function or diaphragm or other respiratory muscle effectiveness) is unclear. We argue that patients in relatively good physical condition who are unable to be weaned from mechanical ventilation might have a potential for improvement through physiotherapy steps.

Furthermore, we found that the weaning outcome was influenced by underlying disease and age, in agreement with previous data from our institution and other studies. ${ }^{21,31}$ These 


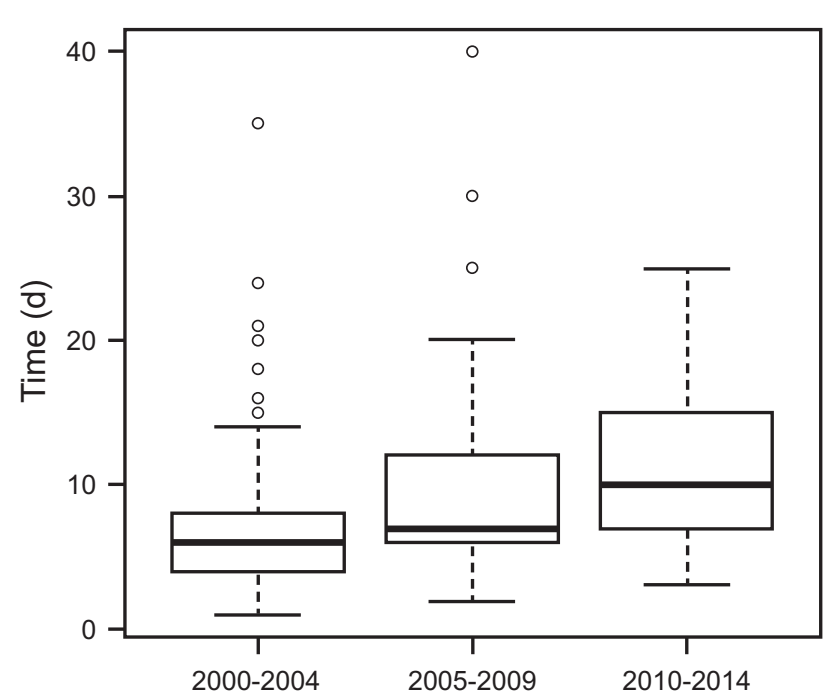

Fig. 3. The median duration of weaning increased in 2005-2009 and 2010-2014, as compared with 2000-2004: 7 (6-12) d vs 6 (4-8) d, $P<.001 ; 10$ (7-15) d vs 6 (4-8) d, $P<.001$, respectively. Center lines denote median, and boxes show 25 th and 75 th percentiles. Points represent outliers.

confounding factors make it difficult if not impossible to draw conclusions regarding the role of physiotherapy in causing weaning success or failure.

Subjects with postsurgical or acute hypoxemic respiratory failure were more likely to be liberated from prolonged mechanical ventilation than were subjects with neuromuscular or chronic pulmonary diseases. This finding is not surprising, given that the cause of postsurgical or acute hypoxemic respiratory failure may represent an intercurrent, and thus potentially reversible, event, whereas most patients with neuromuscular or chronic pulmonary diseases have a disease with a natural evolution toward chronic respiratory failure. These data were confirmed with both univariate and multivariate analyses.

Another finding from the analysis of our population is the achievement of higher physiotherapy steps over the study years despite the decreasing rate of successful weaning over the same period of time. Regarding the achievement of higher physiotherapy steps over the years, we postulate that the expertise and skills of the care team, especially physiotherapists, in treating patients with these characteristics increased significantly. Furthermore, the physiotherapist/patient ratio increased from 0.11 at the beginning of the study to 0.14 in the last period. Finally, the introduction in 2009 of 2 new devices with sophisticated technologies may have further contributed to improvement. The key role of technology in the outcomes of critically ill subjects is also supported by previous data. ${ }^{32}$

In contrast, we observed a decrease in the rate of successful weaning over time, as well as an increase in the time necessary to achieve this goal. Other authors have reported that patients admitted to weaning units are increasingly ill with more comorbidities, and these trends significantly affect the weaning rate. ${ }^{33}$ Our weaning rate was in line with that reported by Polverino and coworkers $^{33}$ during the same period (66\% in 2005) and with those reported by other authors during earlier and later periods. ${ }^{31,34}$ One might argue that the opposite trend between the decreasing weaning rate and the increasing physiotherapy steps achieved is in contrast to our observation of the positive effect of physiotherapy on the process of liberation from mechanical ventilation. However, the trend toward a lower weaning rate may have been due to a change in epidemiology such that ICU survivors became chronically critically ill patients. ${ }^{35}$ Therefore, physiotherapy might have reduced the decrease in weaning success rate. In this light, these 2 trends should be interpreted as separate and reflecting different aspects of the same patient population. Despite the possible objection that subjects with lower physiotherapy steps and failed weaning might be the most ill, this possibility appears to be unlikely, given the homogeneity of severity scores at admission. However, there is no evidence that the SAPS II severity score is a predictable index with regard to improvement in physiotherapy steps or weaning from a mechanical ventilator.

A further explanation of this weaning trend over the years may lie in the significant and progressive increase in the number of patients with neuromuscular diseases (as shown in Fig. 2). As recently shown by Dres et al, ${ }^{36}$ in many subjects, the process of muscular strength recovery may follow separate trajectories in different muscle groups (eg, diaphragm dysfunction is 2-fold more frequent than limb weakness), thus providing a possible further explanation for the dissociation between weaning and peripheral muscular recovery. The mortality rate that we observed is in line with that reported by other authors in similar patient populations. . $^{31,33,34}$

\section{Study Limitations}

Our study has several limitations. First, this study was not a randomized, controlled trial, and as such it cannot assess whether physiotherapy promotes weaning from mechanical ventilation. This study was primarily descriptive and reports data from a single institution; therefore, our findings may not be fully representative of similar trends from other weaning units. These analyses do, however, provide information on results from an actual clinical program. Another limitation is that the absence of a control group undergoing a weaning program without physiotherapy weakens interpretation of the results. However, in light of the existing literature, it would have been unethical to admit patients to a rehabilitation center without performing physiotherapy. Finally, we were unable to perform comparisons of the subjects' conditions prior to mechan- 


\section{Physiotherapy and WeAning From PMV}

ical ventilation. Improving physiotherapy steps might be easy in relatively healthy patients but difficult in patients with paralysis or orthopedic diseases.

\section{Conclusions}

Our data support the inclusion of physiotherapy in the management of patients requiring prolonged mechanical ventilation. Our study also describes the predictive factors of weaning success. Our data confirm that subjects with chronic and progressive diseases impairing respiratory function, as compared with acute events such as postsurgical and acute hypoxemic respiratory failure, have poorer weaning outcomes. Our results should be confirmed by future multi-center, randomized, controlled trials. Research into weaning from prolonged mechanical ventilation in chronic respiratory care is insufficient compared with acute respiratory care. Therefore, these data should be useful for chronic respiratory care and rehabilitation.

\section{REFERENCES}

1. Esteban A, Anzueto A, Frutos F, Alía I, Brochard L, Stewart TE, et al. Characteristics and outcomes in adult patients receiving mechanical ventilation: a 28-day international study. JAMA 2002;287(3): 345-355.

2. Boles JM, Bion J, Connors A, Herridge M, Marsh B, Melot C, et al. Weaning from mechanical ventilation. Eur Respir J 2007;29(5):10331056.

3. McConville JF, Kress JP. Weaning patients from the ventilator. NEngl J Med 2012;367(23):2233-2239.

4. Peñuelas O, Frutos-Vivar F, Fernández C, Anzueto A, Epstein SK, Apezteguía C, et al. Characteristics and outcomes of ventilated patients according to time to liberation from mechanical ventilation. Am J Respir Crit Care Med 2011;184(4):430-437.

5. Bonnici D, Sanctuary T, Warren A, Murphy PB, Steier J, Marino P, et al. Prospective observational cohort study of patients with weaning failure admitted to a specialist weaning, rehabilitation and home mechanical ventilation centre. BMJ Open 2016;6(3):e010025.

6. Nevins ML, Epstein SK. Weaning from prolonged mechanical ventilation. Clin Chest Med 2001;22(1):13-33.

7. Rudy EB, Daly BJ, Douglas S, Montenegro HD, Song R, Dyer MA. Patient outcome for the chronically critically ill: special care unit versus intensive care unit. Nurs Res 1995;44(6):324-331.

8. Seneff MG, Wagner D, Thompson D, Honeycutt C, Silver MR. The impact of long-term acute-care facilities on the outcome and cost of care for patients undergoing prolonged mechanical ventilation. Crit Care Med 2000;28(2):342-350.

9. Scala R, Corrado A, Confalonieri M, Marchese S. Ambrosino N, on behalf of the Scientific Group on Respiratory Intensive Care of the Italian Association of Hospital Pneumologists. Increased number and expertise of Italian respiratory high-dependency care units: the second national survey. Respir Care 2011;56(8):1100-1107.

10. Martin UJ, Hincapie L, Nimchuk M, Gaughan J, Criner GJ. Impact of whole-body rehabilitation in patients receiving chronic mechanical ventilation. Crit Care Med 2005;33(10):2259-2265.

11. Schmidt GA, Girard TD, Kress JP, Morris PE, Ouellette DR, Alhazzani W, et al. Official executive summary of an American Thoracic Society/American College of Chest Physicians clinical practice guideline: liberation from mechanical ventilation in critically ill adults. Am J Respir Crit Care Med 2017;195(1):115-119.
12. Fan E, Cheek F, Chlan L, Gosselink R, Hart N, Herridge MS, et al. An official American Thoracic Society Clinical Practice guideline: the diagnosis of intensive care unit acquired weakness in adults. Am J Respir Crit Care Med 2014;190(12):1437-1446.

13. Schweickert WD, Pohlman MC, Pohlman AS, Nigos C, Pawlik AJ, Esbrook CL, et al. Early physical and occupational therapy in mechanically ventilated, critically ill patients: a randomised controlled trial. Lancet 2009;373(9678):1874-1882.

14. Connolly B, O'Neill B, Salisbury L, Blackwood B. Physical rehabilitation interventions for adult patients during critical illness: an overview of systematic reviews. Thorax 2016;71(10):881-890.

15. Gosselink R, Bott J, Johnson M, Dean E, Nava S, Norrenberg M, et al. Physiotherapy for adult patients with critical illness: recommendations of the European Respiratory Society and European Society of Intensive Care Medicine Task Force on physiotherapy for critically ill patients. Intensive Care Med 2008;34(7):1188-1199.

16. Hanekom S, Gosselink R, Dean E, van Aswegen H, Roos R, Ambrosino N, Louw Q. The development of a clinical management algorithm for early physical activity and mobilization of critically ill patients: synthesis of evidence and expert opinion and its translation into practice. Clin Rehabil 2011;25(9):771-787.

17. Segers J, Hermans G, Bruyninckx F, Meyfroidt G, Langer D, Gosselink R. Feasibility of neuromuscular electrical stimulation in critically ill patients. J Crit Care 2014;29(6):1082-1088.

18. Wright SE, Thomas K, Watson G, Baker C, Bryant A, Chadwick TJ, et al. Intensive versus standard physical rehabilitation therapy in the critically ill (EPICC): a multicentre, parallel-group, randomised controlled trial. Thorax 2018;73(3):213-221.

19. Ambrosino N, Vitacca M. The patient needing prolonged mechanical ventilation: a narrative review. Multidiscip Respir Med 2018;13:6.

20. Nava S. Rehabilitation of patients admitted to a respiratory intensive care unit. Arch Phys Med Rehabil 1998;79(7):849-854.

21. Ceriana C, Delmastro M, Rampulla C, Nava S. Demographics and clinical outcomes of patients admitted to a respiratory intensive care unit located in a rehabilitation center. Respir Care 2003;48(7):670676.

22. Morris P, Goad A, Thompson C, Taylor K, Harry B, Passmore L, et al. Early intensive care unit mobility therapy in the treatment of acute respiratory failure. Crit Care Med 2008;36(8):2238-2243.

23. MacIntyre NR, Epstein SK, Carson S, Scheinhorn D, Christopher K, Muldoon S. Management of patients requiring prolonged mechanical ventilation: report of a NAMDRC consensus conference. Chest 2005; 128(6):3937-3954.

24. Blackwood B, Alderdice F, Burns K, Cardwell C, Lavery G, O'Halloran P. Use of weaning protocols for reducing duration of mechanical ventilation in critically ill adult patients. Cochrane system review and meta-analysis. BMJ 2011;342:c7237.

25. Auriant I, Vinatier I, Thaler F, Tourneur M, Loirat P. Simplified Acute Physiology Score II for measuring severity of illness in intermediate care units. Crit Care Med 1998;26(8):1368-1371.

26. American Medical Association. AMA Guides to the evaluation of permanent impairment, 4th ed. Chicago, IL: American Medical Association; 1995.

27. A language and environment for statistical computing. R Foundation for Statistical Computing, Vienna, Austria. http://www.R-project.org.

28. Brochard L, Thille AW. What is the proper approach to liberating the weak from mechanical ventilation? Crit Care Med 2009;37(Suppl 10):S410-S415

29. Carlucci A, Ceriana P, Prinianakis G, Fanfulla F, Colombo R, Nava $\mathrm{S}$. Determinants of weaning success in patients with prolonged mechanical ventilation. Crit Care 2009;13(3):R97.

30. Martin AD, Smith BK, Davenport PD, Harman E, Gonzalez-Rothi $\mathrm{RJ}, \mathrm{Baz} \mathrm{M}$, et al. Inspiratory muscle strength training improves wean- 


\section{Physiotherapy and WeANing From PMV}

ing outcome in failure to wean patients: a randomized trial. Crit Care 2011;15(2):R84.

31. Davies MG, Quinnell TG, Oscroft NS, Clutterbuck SP, Shneerson JM, Smith IE. Hospital outcomes and long-term survival after referral to a specialized weaning unit. Br J Anaesth 2017;118(84):563569 .

32. Needham DM, Truong AD, Fan E. Technology to enhance physical rehabilitation of critically ill patients. Crit Care Med 2009;37(Suppl 10):S436-S441.

33. Polverino E, Nava S, Ferrer M, Ceriana P, Clini E, Spada E, et al. Patients' characterization, hospital course and clinical outcomes in five Italian respiratory intensive care units. Intensive Care Med 2010; 36(1):137-142.

34. Carpenè N, Vagheggini G, Panait E, Gabbrielli L, Ambrosino N. A proposal of a new model for long-term weaning: respiratory intensive care unit and weaning center. Respir Med 2010;104(10):1505-1511.

35. Lamas D. Chronic critical illness. N Engl J Med 2014;370(2):175-177.

36. Dres M, Dubé BP, Mayaux J, Delemazure J, Reuter D, Brochard L, et al. Coexistence and impact of limb muscle and diaphragm weakness at time of liberation from mechanical ventilation in medical intensive care units patients. Am J Respir Crit Care Med 2017;195(1):57-66.

This article is approved for Continuing Respiratory Care Education credit. For information and to obtain your CRCE

(free to AARC members) visit 